\title{
Electroejaculation and semen analysis and freezing in the giant panda (Ailuropoda melanoleuca)
}

\author{
C. C. Platz, Jr, D. E. Wildt*, J. G. Howard $\dagger$ and M. Bush $\dagger$ \\ Department of Veterinary Physiology and Pharmacology, College of Veterinary Medicine, Texas \\ A \& M University, College Station, Texas 77843; *Section of Genetics, National Cancer Institute, \\ Frederick, Maryland 21701, and $\dagger$ National Zoological Park, Smithsonian Institution, Washington, \\ D.C. 20008 , U.S.A.
}

\begin{abstract}
Summary. Semen was collected by a standardized electroejaculation procedure from a giant panda on 4 occasions. Ejaculate volume, sperm count and $\%$ sperm motility were $2 \cdot 3-3.6 \mathrm{ml}, 62-562 \times 10^{6}$ spermatozoa $/ \mathrm{ml}$ and $45-85 \%$, respectively. The results, although limited to a single male, suggested a seasonal influence on ejaculate and gonadal parameters with improved ejaculate volume, sperm motility and increased testicular size in the season proximate to the female's oestrous period. Frozen-thawed spermatozoa were motile with no apparent abnormalities induced by the freezing procedure.
\end{abstract}

\section{Introduction}

Davis (1964) has described the reproductive anatomy of the male and female giant panda. More recent reports have concerned behavioural alterations associated with oestrus (Kleiman, Karesh \& $\mathrm{Chu}, 1979$ ) and endocrine correlates related to sexual receptivity in the female (Bonney, Wood \& Kleiman, 1981). It is now well established that the female is monoestrous, exhibiting distinct behavioural signs indicative of sexual receptivity for 2-3 days yearly, usually in April or May. These overt oestrous periods can be clearly related to significant elevations in urinary oestrogen secretions. Because of the rarity of the giant panda, little other baseline information on reproductive characteristics exists, particularly for the male.

The National Zoological Park in Washington, D.C., has maintained a pair of giant pandas since 1972. The inability of the female to conceive has generally been considered to be due to behavioural and not physiological dysfunction of the 2 animals. To evaluate reproductive soundness, the male giant panda was anaesthetized on 4 occasions and semen was collected by electroejaculation. Since artificial insemination has been considered a potential alternative to natural mating, the semen was frozen for possible use in future artificial breeding attempts.

\section{Materials and Methods}

At the first semen collection in 1979 the male giant panda, Hsing-Hsing, was 8 years of age, $109 \mathrm{~kg}$ in weight and considered sexually mature. Maintenance and management procedures associated with daily animal care have been described (Melun, 1975). At the time of each electroejaculation, the male was housed separately but was in visual and olfactory proximity to the female.

Anaesthesia was induced and maintained by an i.m. injection of the drug Tilazol (tiletamine- 
$\mathrm{HCl}-Z$ Zlazepam, $100 \mathrm{mg} / \mathrm{ml}$; Parke-Davis, Detroit, Michigan) at an induction dosage of $0.5 \mathrm{mg} / \mathrm{kg}$ body weight. The genital region was cleaned using a sterile saline wash and each testis was measured with a dial micrometer. Testicular size (length $\times$ width) data were converted to volume by the formula for calculating volume of a prolate spheroid (Harrison, Dominque, Heidger, Roberts \& Schlegel, 1977).

The bipolar rectal probe consisted of a rod $56.5 \mathrm{~cm}$ long and $4.5 \mathrm{~cm}$ in diameter. The electrodes, positioned at 30,90 and $150^{\circ}$, were $1.0 \mathrm{~cm}$ in diameter and $15 \mathrm{~cm}$ long. A sterile lubricant was applied to the probe before its insertion $35 \mathrm{~cm}$ into the rectum. The middle electrode was positioned ventrally. The panda was placed in a lateral or dorso-lateral recumbent position during electroejaculation and a plastic specimen cup was placed over the penis to collect the ejaculate. The methodology for electroejaculation was a modification of the procedure previously described in detail (Platz \& Seager, 1978). An a.c. $60 \mathrm{~Hz}$ current was administered using a multimeter stimulator with an isolated current (P-T Electronics, Route 4, Box 338, College Station, Texas 77840). Application of electrical stimuli consisted of a slow steady rise from $0 \mathrm{~V}$ to initial peak voltage over a $3 \mathrm{sec}$ time period. Peak voltage was determined by the physical response of the male using rear leg extension and slight adduction of the hind feet as indicators. The current was held at this peak for $2-3 \mathrm{sec}$ and then returned rapidly to zero. The stimulus was repeated after a $2-3 \mathrm{sec}$ interval at zero. Voltage was increased in increments of $1 \mathrm{~V}$ after administering a series of 10 stimuli at the same voltage or if the male's physical response indicated developing refractoriness.

Ejaculate volume was recorded and microscopic analysis conducted as previously described (Platz, Wildt, Bridges, Seager \& Whitlock, 1980) on sperm morphology, \% motility and progressive (0-5 rating, 5 motility $=$ rapid forward speed of progression). Sperm dimensions using a calibrated eyepiece and photomicroscopy were performed on a fresh wet mount slide at $\times 2000$ on a Zeiss PMIII photomicroscope. For ejaculate preservation, the semen was extended $(1: 2, v / v)$ in a commercial diluent (Life Forces, Inc., P.O. Drawer EK, College Station, Texas 77841) and equilibrated for $90 \mathrm{~min}$ at $5^{\circ} \mathrm{C}$. The extended semen was pelleted onto solid $\mathrm{CO}_{2}$ and then the frozen pellets (vol. $\cong 0.03 \mathrm{ml}$ ) were deposited and stored in liquid nitrogen (Platz, Follis, Demorest \& Seager, 1976; Platz, Wildt \& Seager, 1978). The motility of the spermatozoa was assessed after rapid thawing of 3 pellets in $0.5 \mathrm{ml} 0.85 \%(\mathrm{w} / \mathrm{v}) \mathrm{NaCl}$ at $37^{\circ} \mathrm{C}$.

\section{Results}

Anaesthesia was induced for about $\mathbf{4 0} \mathrm{min}$ at a depth sufficient to perform the electroejaculation procedure. Recovery was uneventful and no adverse post-operative effects were observed.

The electrical stimulation procedure produced ejaculation on each occasion. Voltage and milliamperage ranged from 2 to $10 \mathrm{~V}$ and 100 to $400 \mathrm{~mA}$, respectively. An average of 140 individual stimuli (range 122-150) were provided/electroejaculation procedure.

A summary of the analyses of the raw ejaculates is provided in Table 1.

Table 1. Semen analysis in the giant panda after electroejaculation

\begin{tabular}{|c|c|c|c|c|c|c|c|c|c|}
\hline \multirow[b]{3}{*}{ Date } & \multirow{3}{*}{$\begin{array}{l}\text { Ejaculate } \\
\text { vol. (ml) }\end{array}$} & \multirow{3}{*}{$\begin{array}{c}\text { Total sperr } \\
\text { count } \\
\left(\times 10^{6}\right)\end{array}$} & \multirow{3}{*}{$\begin{array}{c}\% \\
\text { Motility }\end{array}$} & \multirow{3}{*}{$\begin{array}{c}\text { Progressive } \\
\text { motility }\end{array}$} & \multicolumn{5}{|c|}{ Mean \pm s.e.m. sperm dimensions $(\mu \mathrm{m})^{*}$} \\
\hline & & & & & \multicolumn{2}{|c|}{ Head } & \multicolumn{2}{|c|}{ Midpiece } & \multirow{2}{*}{$\begin{array}{l}\text { Flagellum } \\
\text { length }\end{array}$} \\
\hline & & & & & Length & Width & Length & Width & \\
\hline 6 June 1979 & 3.6 & 655 & 75 & 5 & \multirow{4}{*}{\multicolumn{2}{|c|}{$5 \cdot 0 \pm 04 \cdot 2 \pm 0 \cdot 1$}} & $7 \cdot 2 \pm 0 \cdot 12$ & $0.8 \pm 0.04$ & $39.0 \pm 1.33$ \\
\hline 8 June 1979 & $3 \cdot 5$ & 217 & 65 & 5 & & & & & \\
\hline 16 July 1980 & $3 \cdot 5$ & 1967 & 85 & 5 & & & & & \\
\hline 6 May 1980 & $2 \cdot 3$ & 408 & 45 & 3 & & & & & \\
\hline
\end{tabular}

* Measured on fresh mount preparations at $\times 2000$ for 20 random spermatozoa. 
Combined testes volume varied with season of the year but did not vary within the period from 1979 to 1980 (Table 2). Also of interest was the considerable reduction in apparent testicular volume between the June-July and November values. In the latter month, combined testicular volume was only $53.9 \%$ of that measured during July of the same year, which suggests a possible seasonality.

Table 2. Testicular volume in the giant panda

\begin{tabular}{cccc}
\hline \multirow{2}{*}{ Date } & \multicolumn{2}{c}{ Testicular length $\times$ width $(\mathrm{cm})$} & \\
\cline { 2 - 3 } & Right & Left & Volume $(\mathrm{ml})$ \\
\hline 6 June 1979 & $8.9 \times 5.4$ & $9.0 \times 5.7$ & 289.1 \\
16 July 1980 & $9.9 \times 7.5$ & $9.4 \times 7.8$ & 590.5 \\
6 Nov. 1980 & $8.6 \times 5.9$ & $8.9 \times 5.6$ & 317.0 \\
\hline
\end{tabular}

Individual spermatozoa were characterized by a rounded head and an average total length of $51.2 \mu \mathrm{m}$. Abnormal morphological forms constituted $15-30 \%$ of the total spermatozoa and consisted primarily of spermatozoa with bent or coiled tails.

The extender utilized did exhibit a cryoprotective capability. Post-thaw evaluation of frozen pelleted semen gave values of $48 \%$ (range $40-55 \%$ ) motility and $3 \cdot 2$ (range $2 \cdot 5-4.0$ ) for progressive motility. Microscopic analysis of the thawed spermatozoa did not indicate any detectable damage due to freezing.

\section{Discussion}

The results demonstrated that standard electroejaculation procedures can be consistently effective in the giant panda without endangering the health of this rare animal. The sperm count varied considerably for the 4 ejaculates obtained. Such variations have been observed for other animals subjected to electroejaculation but, in the giant panda, may have been the result of ejaculation frequency and season. Three of the 4 collections were obtained during months of the year proximate to the natural breeding season and within 30-60 days of oestrus in the companion female. The June collections were taken $48 \mathrm{~h}$ apart and there was a substantial decline in sperm count but no comparable decrease in ejaculate volume. During the anoestrous period in November, the sperm count was not appreciably different from that of the first collection in June, but ejaculate volume and sperm \% motility and progressive motility tended to be depressed, even though a similar number of electrical stimuli had been applied.

Giant panda spermatozoa have a general shape similar to those of some bears, but total length $(51.2 \mu \mathrm{m})$ was shorter than that recorded for the sloth bear $(72.5 \mu \mathrm{m})$, Kodiak bear $(69.4 \mu \mathrm{m})$ and the spectacled bear $(73 \cdot 1 \mu \mathrm{m})$ (C. C. Platz, unpublished data).

Post-thaw sperm recovery (= post-thaw $\%$ motility $\div$ post-collection $\%$ motility) in the giant panda was $69 \%, 88 \%$ in the European brown bear, $61 \%$ in the spectacled bear and $67 \%$ in the sloth bear. Therefore, sperm recovery appears to be within the range observed for species of bears. At this time comparative evaluations are being conducted at the National Zoo, Washington, D.C., and the National Cancer Institute, Bethesda, Maryland, to determine whether the giant panda is more closely related to raccoons or bears. 


\section{References}

Bonney, R.C., Wood, D.J. \& Kleiman, D.C. (1981) Endocrine correlates of behavioural oestrus in the female giant panda (Ailuropoda melanoleuca) and associated hormonal changes in the male. $J$. Reprod. Fert. 64, 209 215.

Davis, D.D. (1964) The Giant Panda: a Morphological Study of Evolutionary Mechanisms, pp. 221-228. Chicago Natural History Museum, Chicago.

Harrison, R.M., Dominque, G.J., Heidger, P.M., Roberts, J.A. \& Schlegel, J.U. (1977) Vasectomy in rhesus monkeys. I. Surgical techniques and gross observations. Urology 9, 639-644.

Kleiman, D.G., Karesh, W.B. \& Chu, P.R. (1979) Behavioural changes associated with oestrus in the giant panda (Ailuropoda melanoleuca). Int. Zoo $\mathrm{Yb}$. 19, $217-223$.

Melun, N.C. (1975) Giant panda exhibit at the National Zoological Park, Washington. Int. Zoo Yb. 19, 217223 .
Platz, C.C. \& Seager, S. (1978) Semen collection by electroejaculation in the domestic cat. J. Am. Vet. Med. Assoc. 173, 1353-1355.

Platz, C.C., Follis, T., Demorest, N. \& Seager, S. (1976) Semen collection, freezing and insemination in the domestic cat. Proc. 8 th Int Cong. Anim. Reprod. A.I. Kracow, pp. 1053-1056.

Platz, C.C., Wildt, D. \& Seager, S. (1978) Pregnancy in the domestic cat after artificial insemination with previously frozen spermatozoa. J. Reprod. Fert. 52, 279-282.

Platz, C.C., Wildt, D., Bridges, C., Seager, S. \& Whitlock, B. (1980) Electroejaculation and semen analysis in a male lowland gorilla, Gorilla gorilla gorilla. Primates 21, 130-132.

Received 18 January 1982 